\title{
Integrating multiple microarray dataset analysis and machine learning methods to reveal the key genes and regulatory mechanism s underlying human intervertebral disc degeneration
}

\author{
Hongze Chang ${ }^{1}$, Xiaolong Yang ${ }^{1}$, Kemin You ${ }^{1}$, Mingwei Jiang ${ }^{1}$, Feng Cai $^{1}$, Yan Zhang ${ }^{1}$, Liang Liu $^{1}$, Hui Liu $^{1}$, \\ Xiaodong Liu Corresp. 1 \\ ${ }^{1}$ Department of orthopedics, Shanghai Yangpu Hospital Affiliated to Tongji University, Shanghai, China \\ Corresponding Author: Xiaodong Liu \\ Email address: xiaodong.liu@tongji.edu.cn
}

Intervertebral disc degeneration (IDD), a major cause of lower back pain, has multiple contributing factors including genetics, environment, age, and loading history.

Bioinformatics analysis has been extensively used to identify diagnostic biomarkers and therapeutic targets for IDD diagnosis and treatment. However, multiple microarray dataset analysis and machine learning methods have not been integrated. In this study, we downloaded the mRNA, microRNA (miRNA), long noncoding RNA (InCRNA), and circular RNA (circRNA) expression profiles (GSE34095, GSE15227, GSE63492, GSE116726, GSE56081, and GSE67566) associated with IDD from the GEO database. Using differential expression analysis and recursive feature elimination, we extracted four optimal feature genes. We then used the support vector machine (SVM) to make a classification model with the four optimal feature genes. The ROC curve was used to evaluate the model's performance, and the expression profiles (GSE63492, GSE116726, GSE56081, and GSE67566) were used to construct a competitive endogenous RNA (ceRNA) regulatory network and explore the underlying mechanisms of the feature genes. We found that three miRNAs (hsa-miR-4728-5p, hsa-miR-5196-5p, and hsa-miR-185-5p) and three circRNAs (hsa_circRNA_100723, hsa_circRNA_104471, and hsa_circRNA_100750) were important regulators with more interactions than the other RNAs across the whole network. The expression level analysis of the three datasets revealed that BCAS4 and SCRG1 were key genes involved in IDD development. Ultimately, our study proposes a novel approach to determining reliable and effective targets in IDD diagnosis and treatment. 
1 Integrating multiple microarray dataset analysis and machine learning methods to reveal 2 the key genes and regulatory mechanisms underlying human intervertebral disc 3 degeneration

4

5 Hongze Chang, Xiaolong Yang, Kemin You, Mingwei Jiang, Feng Cai, Yan Zhang, Liang Liu, Hui Liu, 6 Xiaodong Liu*

8 Department of Orthopedics, Shanghai Yangpu Hospital Affiliated to Tongji University, 450 Tengyue Road, 9 Shanghai 200090, China

* Corresponding author:

12 Xiaodong Liu (xiaodong.liu@tongji.edu.cn)

13 Department of Orthopedics, Shanghai Yangpu Hospital Affiliated to Tongji University, 450 Tengyue Road, 14 Shanghai 200090, China

Running title: Integrated analysis and machine learning methods reveal key genes and underlying mechanisms underlying IDD. 


\begin{abstract}
:
Intervertebral disc degeneration (IDD), a major cause of lower back pain, has multiple contributing factors including genetics, environment, age, and loading history. Bioinformatics analysis has been extensively used to identify diagnostic biomarkers and therapeutic targets for IDD diagnosis and treatment. However, multiple microarray dataset analysis and machine learning methods have not been integrated. In this study, we downloaded the mRNA, microRNA (miRNA), long noncoding RNA (lncRNA), and circular RNA (circRNA) expression profiles (GSE34095, GSE15227, GSE63492, GSE116726, GSE56081, and GSE67566) associated with IDD from the GEO database. Using differential expression analysis and recursive feature elimination, we extracted four optimal feature genes. We then used the support vector machine (SVM) to make a classification model with the four optimal feature genes. The ROC curve was used to evaluate the model's performance, and the expression profiles (GSE63492, GSE116726, GSE56081, and GSE67566) were used to construct a competitive endogenous RNA (ceRNA) regulatory network and explore the underlying mechanisms of the feature genes. We found that three miRNAs (hsa-miR-4728-5p, hsa-miR-5196-5p, and hsa-miR-185-5p) and three circRNAs (hsa_circRNA_100723, hsa_circRNA_104471, and hsa_circRNA_100750) were important regulators with more interactions than the other RNAs across the whole network. The expression level analysis of the three datasets revealed that $B C A S 4$ and $S C R G 1$ were key genes involved in IDD development. Ultimately, our study proposes a novel approach to determining reliable and effective targets in IDD diagnosis and treatment.
\end{abstract}

\title{
Keywords:
}

Intervertebral disc degeneration; Integrated analysis; Machine learning methods; Key genes

\section{Introduction}

Lower back pain, commonly caused by intervertebral disc degeneration (IDD), can be a significant socioeconomic burden on patients (Vergroesen et al. 2015). IDD is characterized by the apoptosis of nucleus pulposus (NP) cells, the degradation of extracellular matrix (ECM) components, and several contributing factors including genetics and environment (Battie et al. 2008; Feng et al. 2016). However, the precise etiology of IDD remains largely unknown. Diagnosing degenerative disc disease is difficult, and common IDD treatment and management strategies primarily consist of conservative management or surgical treatment to relieve pain, without resolving the underlying tissue pathology (An et al. 2003; Zaina et al. 2016). To identify potential biomarkers and specific therapeutic targets, a more detailed understanding of the molecular and cellular events underlying IDD formation is needed.

The etiology of IDD is complex, but over the past several decades, it has become clear that genetic factors are most dominant. Multiple candidate genes, including thrombospondin-2, vitamin D receptor, COL2A1, ACAN, interleukins ( $I L 1 \alpha, I L 1 \beta$, and $I L \sigma$ ), matrix metalloproteinases (MMP-3 and MMP-9), and growth/differentiation factor 5, have been associated with the pathophysiological process of IDD development (Feng et al. 2016; Kalb et al. 2012; Wang et al. 2018b; Yuan et al. 2018). Genome-wide association studies (GWAS) have been used to help identify novel variants. Several GWAS found that the genetic polymorphisms of PARK2 and CHST3 were 
relevant to IDD etiology (Song et al. 2013; Williams et al. 2013). Additionally, some bioinformatics analyses based on gene expression profiles revealed that FYN, PRKCD, YWHAB, YWHAZ, AR, Fibronectin 1, COL2A1, $\beta$-catenin, COL6A2, IBSP, RAP1A, and FOXF2 genes may play key roles in IDD development (Chen et al. 2013; Guo et al. 2017; Ji et al. 2015). Although a considerable number of genes associated with IDD development have been found, early IDD diagnosis and precise treatment remain difficult and require further study.

Integrated analyses using combined multiple microarray data can provide a more accurate understanding of the interplay across multi-level genomic features and the molecular mechanisms that cause complex diseases (Momtaz et al. 2018). Machine learning is a type of artificial intelligence that can "learn" a model using past data in order to predict future data. Machine learning algorithms have been used in key feature training, recognition, and group classification (Huang et al. 2018). Modern researchers have unprecedented access to machine learning methods that can elucidate complex molecular mechanisms and predict disease genes from large biomedical datasets (Libbrecht \& Noble 2015; Obermeyer \& Emanuel 2016). To the best of our knowledge, no investigations have used integrated analysis and machine learning methods to identify key IDD-associated genes.

Accumulating evidence has indicated that noncoding RNAs, including microRNAs (miRNAs), long noncoding RNAs (lncRNAs), and circular RNAs (circRNAs), are important gene expression regulators that influence cellular function and disease states (Adams et al. 2017). lncRNAs and circRNAs act as competitive endogenous RNAs (ceRNAs) and competitively bind miRNA response elements (MREs) to construct a regulatory network involved in IDD progression. Zhao et al. (2016) used RNA sequencing to identify 1,854 lncRNAs and 2,804 protein-coding genes that were differentially expressed in the IDD group. Tan et al. (2018) found that LncSNHG1 promoted NP cell proliferation by suppressing miR-326 expression and upregulating CCND1 expression. Recent studies have focused on the functional roles of circRNAs during IDD development and found that circ-4099, circ-GRB10, circVMA21, and circ_001653 play pivotal roles during NP cell proliferation, apoptosis, and extracellular matrix synthesis/degradation (Cheng et al. 2018; Cui \& Zhang 2020; Guo et al. 2018; Wang et al. 2018a). The diverse biological functions of ceRNAs deserve further exploration.

In this study, we aimed to identify the key genes and underlying mechanisms of IDD development by constructing an IncRNA/circRNA-miRNA-mRNA network using multiple microarray datasets and machine learning methods. Fig. S1 shows the flow chart for this study. Our results present novel biomarkers and therapeutic targets that can be used for IDD diagnosis and treatment.

\section{Materials \& Methods}

\section{Microarray datasets}

We retrieved a total of six expression profiles from the GEO database (www.ncbi.Nlm.nih.gov/geo): two mRNA expression profiles (GSE34095 (Tsai et al. 2013) and GSE15227 (Gruber et al. 2009)), two miRNA expression profiles (GSE63492 (Lan et al. 2016) and GSE116726 (Ji et al. 2018)), one mRNA-lncRNA expression profile (GSE56081 (Lan et al. 2016; Wan et al. 2014)), and one circRNA expression profile (GSE67566 (Lan et al. 2016; Liu et al. 2015)). Table 1 contains the basic information for these expression profiles. 


\section{Differential expression analysis}

The raw data were annotated, normalized, $\log 2$ transformed, and screened for differentially expressed genes (DEGs), differentially expressed miRNAs (DEMs), differentially expressed lncRNAs (DELs), and differentially expressed circRNAs (DECs) in IDD and normal disc tissue using the "Limma" R package (Ritchie et al. 2015). Since each dataset came from a different experiment and microarray platform, the way to get the data may be different. When filtering using consistent thresholds, some datasets did not yield valid differential genes. In order to obtain effective differentially expressed genes for subsequent analysis, we used different thresholds according to each dataset's conditions. The specific DEG screening thresholds were as follows: $p$-value $<0.05$ for the GSE34095 dataset, $p$-value $<0.01$ and $|\log 2 \mathrm{FC}| \geq 2$ for the GSE15227 dataset, and p-value $<0.01$ and $|\log 2 \mathrm{FC}|$ $>2$ for the GSE1 16726 dataset. We obtained the GSE67566, GSE63492, and GSE56081 datasets from the same tissue samples, and provided the differential expression analysis results and thresholds as Supplemental Materials (Tables S1 and S2, Documents S1 and S2). We visualized the differential expression analysis results using a volcano plot and heatmap with hierarchical clustering (Fig. S2).

\section{Feature gene extraction}

Using VENNY 2.1 software (http://bioinfogp.cnb.csic.es/tools/venny/index.html), we extracted the differential gene intersections of the GSE34095 and GSE15227 datasets to use as IDD-related DEGs(Li et al. 2019; Zhang et al. 2018). We used the GSE15227 dataset as the training set to screen for important feature genes. Using the $\mathrm{R}$ caret package, random forest, and neural network methods, we constructed the model and obtained the important DEG features (Kuhn 2015). We used recursive feature elimination (RFE), a machine learning method, to extract the optimum feature genes to identify the functional biomarkers involved in IDD progression (Guyon et al. 2002).

\section{SVM model verification}

The support vector machine (SVM) is particularly effective for binary classification in a supervised learning manner, and is better than other machine learning methods at identifying subtle patterns in complex datasets (Aruna \& Dr 2011). Radial basis function kernel (RBF kernel) is commonly used in nonlinear support vector machine classification since it can enable data to operate in a high-dimensional and implicit feature space (Jiao et al. 2017). In this study, we used the R e1071 package (https://CRAN.R-project.org/package=e1071) to build an RBF kernel SVM model to identify the optimal feature genes (Dimitriadou et al. 2012), and we selected the GSE15227 dataset to train the machine. We then evaluated its performance in the GSE15227 training set using the R pROC package (http://www.biomedcentral.com/1471-2105/12/77/) (Robin et al. 2011). The optimal feature genes were taken from the training set, and over-fitting would occur whenever ROC verification was performed. Therefore, we used two validation sets (GSE34095 and GSE56081) to further verify the model's performance.

\section{Identifying target miRNAs of the optimal feature genes}

We used miRWalk 2.0 with 12 prediction programs (MIMATid, Microt4, miRanda, mirbridge, miRDB, miRMap, miRNAMap, Pictar2, PITA, RNA22, RNAhybrid, and Targetscan) to predict the optimal feature 
genes' target miRNAs (Dweep \& Gretz 2015). miRNAs present in more than five of the 12 prediction programs were considered as target miRNAs. We selected overlapping DEMs in the GSE116726 and GSE63492 datasets as candidate DEMs. Intersecting target miRNAs and candidate DEMs were selected as optimal feature gene interaction pairs.

\section{ceRNA network construction}

We obtained the miRNA sequences interacting with optimal feature genes from the miRbase (Kozomara \& Griffiths-Jones 2011) and extracted mature sequences using the Perl program. The DEL sequences were downloaded from NCBI. For DELs with several transcripts, we selected the longest transcripts for subsequent analysis. We used the BEDTOOLS command (Quinlan \& Hall 2010) and genomic coordinates to obtain the DEC sequences, and converted the gene name to its circRNA symbol using the Perl program.

We then used the miRanda tool to analyze the combinations of miRNAs, DELs, and DECs. We set the analysis parameters for the miRNA-DELs and miRNA-DECs as sc:120, en:-20 and sc:150, en:-7, respectively, and processed the results using python script (Documents S3 and S4). The DELs and DECs with $\geq 5$ miRNA binding sites were identified as reliable miRNA-lncRNA and miRNA-circRNA interaction pairs. The ceRNA (DELs/DECs-miRNA-optimal feature gene) regulatory network was constructed using a combination of miRNA-DEL pairs, miRNA-DEC pairs, and miRNA-optimal feature gene pairs. We visualized the network using Cytoscape 3.6.0 (http://www.cytoscape.org/).

\section{Statistical analysis}

All data were presented as the mean \pm SEM. Differential expression levels were compared using the Student's ttest in GraphPad Prism 7.0 (GraphPad Software Inc., La Jolla, CA, USA). P values $<0.05$ were considered statistically significant.

\section{Results}

\section{Differentially expressed genes in IDD}

We obtained a total of 334 DEGs (199 up-regulated and 135 down-regulated) in the GSE34095 dataset (Fig. 1A) and 188 DEGs (141 up-regulated and 47 down-regulated) in the GSE15227 dataset (Fig. 1B). Hierarchical cluster heatmaps showed that these DEGs could distinguish between the degenerative disc samples and the control disc samples (Fig. 1C and 1D). We obtained a total of 13 overlapping DEGs (COL3A1, SCRG1, HTRA1, BCAS4, C11 1orf80, CRNKL1, GREM1, FGFR3, BDKRB1, WDR46, FN1, LMF2, and GDI2) via the intersection between the two datasets, and considered these as IDD-related DEGs (Fig. 1E).

\section{Optimal feature gene selection}

We used random forest and neural network models to evaluate the importance of the 13 overlapping DEGs (Documents S5 and S6). The results showed that most of the DEGs from the two models had minimal differences in terms of gene importance rankings (Fig. 2A and 2B). Using the RFE method, we identified four important genes (WDR46, BCAS4, CRNKL1, and SCRG1) as optimal feature genes associated with IDD (Fig. 2C). 
206

207

208

209

210

211

212

213

214

215

216

217

218

219

220

221

222

223

224

225

226

227

228

229

230

231

232

233

234

235

236

237

238

239

240

241

242

243

244

245

246

A classification model was constructed using the RBF kernel SVM, the four genes as features, and the GSE15227 dataset to train the machine. The parameters were set as: SVM-Kernel: radial, cost:1, gamma:0.25, and epsilon:0.1. Additionally, their performance was assessed using the R pROC package. In the GSE15227 training set, the area under the ROC curve (AUC) of the SVM model was 100 percent, suggesting that the model could accurately distinguish between IDD and normal samples (Fig. 2D). We used the GSE34095 and GSE56081 datasets as validation sets to further evaluate the model's performance and to help avoid over-fitting in the training set. The SVM model in the GSE34095 validation set had an AUC of 55.6 percent, which may be correlated with the small sample size of the microarray dataset (Fig. 2E). However, the AUC for the GSE56081 validation set was 100 percent (Fig. 2F). These results indicated that the optimal feature genes (WDR46, BCAS4, $C R N K L 1$, and SCRG1) could be used as effective and accurate IDD diagnostic biomarkers.

\section{Identifying target miRNAs of the optimal feature genes}

We predicted a total of 467 target miRNA optimal feature genes using miRWalk 2.0. Compared to the control disc samples, we identified 724 DEMs (527 up-regulated and 197 down-regulated) from the GSE116726 dataset (Fig. 3A and 3B) and 149 DEMs from the GSE63492 dataset. Fig. 3C shows a Venn diagram of the 46 common DEMs that were found. Based on the intersections between the target miRNAs and common DEMs, we further analyzed 12 overlapping miRNAs which we considered to be the optimal feature genes' interaction miRNAs (Fig. 3D).

\section{ceRNA network construction}

The differentially expressed lncRNAs and circRNAs between the degenerated disc samples and the control disc samples were downloaded from the GEO database. We analyzed the miRNA-DEL interactions and the miRNADEC interactions using the miRanda tool with $\geq 5$ miRNA binding sites. The miRNA-DEL pairs, miRNA-DEC pairs, and miRNA-optimal feature gene pairs were combined to build a DELs/DECs-DEMs-optimal feature gene regulatory network, which included four mRNA nodes, 12 miRNA nodes, 10 lncRNA nodes, and 75 circRNA nodes (Fig. 4, Table S3). Out of these, three miRNAs (hsa-miR-4728-5p, hsa-miR-5196-5p, and hsa-miR-1855p) and three circRNAs (hsa_circRNA_100723, hsa_circRNA_104471, and hsa_circRNA_100750) were key regulators, based on the optimal feature genes and connective degrees of the whole network (Table 2). However, the LncRNA connective degree was very low.

\section{Validating optimal feature genes}

The expression of the four optimal feature genes across the three datasets was visualized using box plots. In the GSE15227 and GSE56081 datasets, BCAS4 expression levels were significantly down-regulated (Fig.5A and $5 \mathrm{C}$ ), and $S C R G 1$ was significantly up-regulated (Fig. 5G and 5I). CRNKL1 was only up-regulated in the GSE34095 dataset (Fig. 5E). Unfortunately, the expression levels of WDR46 showed no significant difference across the three datasets (Fig. 5J,5K and 5L). These results indicated that BCAS4 and SCRG1 are key genes involved in IDD development.

\section{Discussion}

IDD is linked to lower back pain and spine-related diseases, and although IDD's underlying mechanisms have 
been studied for many years, they still remain unclear. Insufficient early IDD diagnosis and treatment methods affect the quality of life for patients and impose a heavy economic burden on society (Vergroesen et al. 2015). NP cells play an important role in maintaining intervertebral disc homeostasis by synthesizing ECM, which includes aggrecan and type II collagen (Zhang et al. 2016). Recent studies show that targeting gene therapy can inhibit NP cell senescence and apoptosis, and can ultimately ameliorate IDD (Chen et al. 2018). Therefore, reliable and specific gene targets are essential for IDD diagnosis and treatment.

Recent developments in bioinformatics and computational biology have led to the identification of several key genetic targets related to IDD and the prediction of potential molecular mechanisms (Petryszak et al. 2014). In this study, we downloaded multiple microarray datasets associated with IDD from the GEO database, including two mRNA expression profiles, two miRNA expression profiles, one mRNA-lncRNA expression profile, and one circRNA expression profile. A total of four optimal feature genes (WDR46, BCAS4, CRNKL1, and SCRG1) were identified using machine-learning methods. The construction of a DELs/DECs-miRNA-optimal feature genes network revealed that three miRNAs (hsa-miR-4728-5p, hsa-miR-5196-5p, and hsa-miR-185-5p) and three circRNAs (hsa_circRNA_100723, hsa_circRNA_104471, and hsa_circRNA_100750) may be important mediators for optimal feature genes. To further explore the different optimal feature genes in normal and degenerative NP tissues, we also investigated their expression levels across three datasets. The results indicated that $B C A S 4$ and $S C R G 1$ were key genes related to IDD.

Breast carcinoma amplified sequence 4 (BCAS4), a novel gene cloned from breast cancer cells, encodes a 211amino acid cytoplasmic protein with no significant homologies to any known protein (Barlund et al. 2002). Previous studies have demonstrated that the specific DNA methylation of BCAS4 acts as an epigenetic marker and can be used to distinguish saliva from other body fluids. It is also widely used in forensic investigations (Silva et al. 2016; Taki \& Kibayashi 2015). However, BCAS4's biological role in disease requires further investigation. Stimulator of chondrogenesis 1 (SCRG1) was first found in the genes associated with, or responsible for, the neurodegenerative changes observed in transmissible spongiform encephalopathies (Dandoy-Dron et al. 1998). SCRG1 transcript is found in the brain, heart, and spinal cord, and its sequence is highly conserved in humans, mice, and rats. SCRG1 has also been observed to be specifically expressed in human articular cartilage, and is involved in human mesenchymal stem cell (hMSC) growth suppression and differentiation during dexamethasone-dependent chondrogenesis (Ochi et al. 2006). Recent studies have shown that $S C R G 1$ is an important regulator during hMSC self-renewal, migration, and osteogenic differentiation along with its receptor BST1 (Aomatsu et al. 2014). However, the function of SCRG1 in IDD development has not yet been explored.

circRNAs and lncRNAs may act as ceRNAs by competitively binding to miRNA and suppressing mRNA expression (Adams et al. 2017; Tay et al. 2014). This ceRNA hypothesis suggests that there is a novel mechanism for RNA interactions. In this study's ceRNA analysis, hsa-miR-4728-5p, hsa-miR-5196-5p, hsa-miR-185-5p, hsa_circRNA_100723, hsa_circRNA_104471, and hsa_circRNA_100750 showed more interactions compared to the other RNAs in the whole network. Additionally, miR-155, miR-21, and miR-133a were shown to be differentially expressed in degenerative NP cells, indicating that they may be potential biomarkers for early IDD diagnosis (Liu et al. 2014; Wang et al. 2011; Xu et al. 2016). The dysregulation of these miRNAs is closely 
associated with NP cell apoptosis which affects IDD progression. miR-185-5p has been reported as a critical regulator, but miR-4728-5p and miR-5196-5p have rarely been reported. Chang et al. (2017) reported that miR$185-5 \mathrm{p}$ induced by Runx 2 could directly target Dlx2 to inhibit amylogenesis and osteogenesis, providing a new treatment option for cleidocranial dysplasia. Multiple $\operatorname{lncRNAs}$ bind to miR-185-5p in order to modulate the progression of different types of human cancer, including prostate cancer (Tian et al. 2018), colorectal cancer (Zhu et al. 2018), and glioblastoma (Wang et al. 2018b). However, the regulatory effects of miR-185-5p on IDD require further investigation.

CircRNAs, a new type of ncRNAs formed by special loop splicing, are thought to be potential diagnostic biomarkers and therapeutic targets because they are more stable and conserved than other RNAs (Lee et al. 2019). However, very few studies have focused on the role of circRNAs in IDD development. Guo et al. (2018) found that circ-GRB10 was downregulated in IDD cells, and circ-GRB10 overexpression inhibited NP cell apoptosis by sequestering miR-328-5p and upregulating target genes involved in cell proliferation through the ErbB pathway. Cheng et al. (2018) reported that circVMA21 acted as a sponge for miR-200c and regulated the activity and function of NP cells by targeting miRNA-200c and XIAP, providing a new IDD intervention and treatment strategy. Wang et al. (2018a) found that circRNA_4099 could act as a sponge for miR-616-5p and eliminate Sox9 inhibition, increasing ECM secretion. Similarly, Cui \& Zhang (2020) reported that circ_001653 silencing may bind to miR-486-3p in order to inhibit CEMIP expression, thus attenuating NP cell apoptosis and ECM degradation. In this study, we used integrated analysis to identify that hsa_circRNA_100723, hsa_circRNA_104471, and hsa_circRNA_100750, which have not been previously reported, are more likely to be important molecules involved in IDD regulation, and require further investigation.

\section{Conclusion}

In this study, we used integrated bioinformatics analysis and machine learning methods to identify $B C A S 4$ and $S C R G 1$ as key genes associated with IDD development. Additionally, after constructing the ceRNA network, we found three miRNAs and three circRNAs that may act as important regulators during IDD development by targeting key genes. This novel study may provide new insights into IDD pathogenesis and therapy. Further experiments should be conducted to verify this study's results.

\section{Acknowledgements}

Not applicable.

\section{Funding}

This work was supported by Shanghai Natural Science Foundation(NO.20ZR1452400).

\section{Disclosures}

The authors have no financial conflicts of interest.

\section{Availability of data}

The datasets used and/or analyzed in the present study are available from the public Gene Expression Omnibus 
329

330

331

332

333

334

335

336

337

338

339

340

341

342

343

344

345

346

347

348

349

350

351

352

353

354

355

356

357

358

359

360

361

362

363

364

365

366

367

368

369

(GEO) database.

\section{References}

Adams BD, Parsons C, Walker L, Zhang WC, and Slack FJ. 2017. Targeting noncoding RNAs in disease. J Clin Invest 127:761-771. 10.1172/jci84424

An H, Boden SD, Kang J, Sandhu HS, Abdu W, and Weinstein J. 2003. Summary statement: emerging techniques for treatment of degenerative lumbar disc disease. Spine (Phila Pa 1976) 28:S24-25. 10.1097/01.brs.0000076894.33269.19

Aomatsu E, Takahashi N, Sawada S, Okubo N, Hasegawa T, Taira M, Miura H, Ishisaki A, and Chosa N. 2014. Novel SCRG1/BST1 axis regulates self-renewal, migration, and osteogenic differentiation potential in mesenchymal stem cells. Sci Rep 4:3652. 10.1038/srep03652

Aruna S, and Dr S. 2011. A Novel SVM based CSSFFS Feature Selection Algorithm for Detecting Breast Cancer. International Journal of Computer Applications 31:14-20.

Barlund M, Monni O, Weaver JD, Kauraniemi P, Sauter G, Heiskanen M, Kallioniemi OP, and Kallioniemi A. 2002. Cloning of BCAS3 (17q23) and BCAS4 (20q13) genes that undergo amplification, overexpression, and fusion in breast cancer. Genes Chromosomes Cancer 35:311-317. 10.1002/gcc.10121

Battie MC, Videman T, Levalahti E, Gill K, and Kaprio J. 2008. Genetic and environmental effects on disc degeneration by phenotype and spinal level: a multivariate twin study. Spine (Phila Pa 1976) 33:2801-2808. 10.1097/BRS.0b013e31818043b7

Chen J, Xie JJ, Jin MY, Gu YT, Wu CC, Guo WJ, Yan YZ, Zhang ZJ, Wang JL, Zhang XL, Lin Y, Sun JL, Zhu GH, Wang XY, and Wu YS. 2018. Sirt6 overexpression suppresses senescence and apoptosis of nucleus pulposus cells by inducing autophagy in a model of intervertebral disc degeneration. Cell Death Dis 9:56. 10.1038/s41419-017-0085-5

Chen K, Wu D, Zhu X, Ni H, Wei X, Mao N, Xie Y, Niu Y, and Li M. 2013. Gene expression profile analysis of human intervertebral disc degeneration. Genet Mol Biol 36:448-454. 10.1590/s1415-47572013000300021

Cheng X, Zhang L, Zhang K, Zhang G, Hu Y, Sun X, Zhao C, Li H, Li YM, and Zhao J. 2018. Circular RNA VMA21 protects against intervertebral disc degeneration through targeting miR-200c and X linked inhibitor-ofapoptosis protein. Ann Rheum Dis 77:770-779. 10.1136/annrheumdis-2017-212056

Cui S, and Zhang L. 2020. circ_001653 Silencing Promotes the Proliferation and ECM Synthesis of NPCs in IDD by Downregulating miR-486-3p-Mediated CEMIP. Mol Ther Nucleic Acids 20:385-399. 10.1016/j.omtn.2020.01.026

Dandoy-Dron F, Guillo F, Benboudjema L, Deslys JP, Lasmezas C, Dormont D, Tovey MG, and Dron M. 1998. Gene expression in scrapie. Cloning of a new scrapie-responsive gene and the identification of increased levels of seven other mRNA transcripts. J Biol Chem 273:7691-7697. 10.1074/jbc.273.13.7691

Dimitriadou E, Hornik K, Leisch F, Meyer D, and Weingessel A. 2012. Misc functions of the Department of Statistics 
(e1071), TU Wien.

Dweep H, and Gretz N. 2015. miRWalk2.0: a comprehensive atlas of microRNA-target interactions. Nat Methods 12:697. 10.1038/nmeth.3485

Feng Y, Egan B, and Wang J. 2016. Genetic Factors in Intervertebral Disc Degeneration. Genes Dis 3:178-185. 10.1016/j.gendis.2016.04.005

Gruber HE, Hoelscher G, Loeffler B, Chow Y, Ingram JA, Halligan W, and Hanley EN, Jr. 2009. Prostaglandin E1 and misoprostol increase epidermal growth factor production in 3D-cultured human annulus cells. Spine $J$ 9:760-766. 10.1016/j.spinee.2009.04.024

Guo W, Zhang B, Li Y, Duan HQ, Sun C, Xu YQ, and Feng SQ. 2017. Gene expression profile identifies potential biomarkers for human intervertebral disc degeneration. Mol Med Rep 16:8665-8672. 10.3892/mmr.2017.7741

Guo W, Zhang B, Mu K, Feng SQ, Dong ZY, Ning GZ, Li HR, Liu S, Zhao L, Li Y, Yu BB, Duan HQ, Sun C, and Li YJ. 2018. Circular RNA GRB10 as a competitive endogenous RNA regulating nucleus pulposus cells death in degenerative intervertebral disk. Cell Death Dis 9:319. 10.1038/s41419-017-0232-z

Guyon I, Weston J, Barnhill S, and Vapnik V. 2002. Gene Selection for Cancer Classification using Support Vector Machines. Machine Learning 46:389-422.

Huang S, Cai N, Pacheco PP, Narrandes S, Wang Y, and Xu W. 2018. Applications of Support Vector Machine (SVM) Learning in Cancer Genomics. Cancer Genomics Proteomics 15:41-51. 10.21873/cgp.20063

Ji ML, Jiang H, Zhang XJ, Shi PL, Li C, Wu H, Wu XT, Wang YT, Wang C, and Lu J. 2018. Preclinical development of a microRNA-based therapy for intervertebral disc degeneration. Nat Commun 9:5051. 10.1038/s41467018-07360-1

Ji SC, Han N, Liu Y, Li G, Sun Z, and Li Z. 2015. Identification of genes associated with disc degeneration using bioinformatics. Biotech Histochem 90:353-360. 10.3109/10520295.2015.1007481

Jiao P, Cai F, Feng Y, and Wang W. 2017. Link predication based on matrix factorization by fusion of multi class organizations of the network. Scientific reports 7:8937-8937. 10.1038/s41598-017-09081-9

Kalb S, Martirosyan NL, Kalani MY, Broc GG, and Theodore N. 2012. Genetics of the degenerated intervertebral disc. World Neurosurg 77:491-501. 10.1016/j.wneu.2011.07.014

Kozomara A, and Griffiths-Jones S. 2011. miRBase: integrating microRNA annotation and deep-sequencing data. Nucleic Acids Res 39:D152-157. 10.1093/nar/gkq1027

Kuhn M. 2015. Caret: Classification and regression training. Astrophysics Source Code Library 129:291-295.

Lan PH, Liu ZH, Pei YJ, Wu ZG, Yu Y, Yang YF, Liu X, Che L, Ma CJ, Xie YK, Hu QJ, Wan ZY, and Wang HQ. 2016. Landscape of RNAs in human lumbar disc degeneration. Oncotarget 7:63166-63176. 10.18632/oncotarget.11334

Lee ECS, Elhassan SAM, Lim GPL, Kok WH, Tan SW, Leong EN, Tan SH, Chan EWL, Bhattamisra SK, Rajendran R, and Candasamy M. 2019. The roles of circular RNAs in human development and diseases. Biomed Pharmacother 111:198-208. 10.1016/j.biopha.2018.12.052

Li CY, Zhang WW, Xiang JL, Wang XH, Wang JL, and Li J. 2019. Integrated analysis highlights multiple long non-coding RNAs and their potential roles in the progression of human esophageal squamous cell carcinoma. Oncol Rep 42:2583-2599. 10.3892/or.2019.7377

Libbrecht MW, and Noble WS. 2015. Machine learning applications in genetics and genomics. Nat Rev Genet 16:321332. $10.1038 / \mathrm{nrg} 3920$ 
411

412

413

414

415

416

417

418

419

420

421

422

423

424

425

426

427

428

429

430

431

432

433

434

435

436

437

438

439

440

441

442

443

444

445

446

447

448

449

450

451

Liu H, Huang X, Liu X, Xiao S, Zhang Y, Xiang T, Shen X, Wang G, and Sheng B. 2014. miR-21 promotes human nucleus pulposus cell proliferation through PTEN/AKT signaling. Int $J$ Mol Sci 15:4007-4018. 10.3390/ijms 15034007

Liu X, Che L, Xie YK, Hu QJ, Ma CJ, Pei YJ, Wu ZG, Liu ZH, Fan LY, and Wang HQ. 2015. Noncoding RNAs in human intervertebral disc degeneration: An integrated microarray study. Genom Data 5:80-81. 10.1016/j.gdata.2015.05.027

Momtaz R, Ghanem NM, El-Makky NM, and Ismail MA. 2018. Integrated analysis of SNP, CNV and gene expression data in genetic association studies. Clin Genet 93:557-566. 10.1111/cge.13092

Obermeyer Z, and Emanuel EJ. 2016. Predicting the Future - Big Data, Machine Learning, and Clinical Medicine. New England Journal of Medicine 375:1216-1219. 10.1056/NEJMp1606181

Ochi K, Derfoul A, and Tuan RS. 2006. A predominantly articular cartilage-associated gene, SCRG1, is induced by glucocorticoid and stimulates chondrogenesis in vitro. Osteoarthritis and Cartilage 14:30-38. https://doi.org/10.1016/j.joca.2005.07.015

Petryszak R, Burdett T, Fiorelli B, Fonseca NA, Gonzalez-Porta M, Hastings E, Huber W, Jupp S, Keays M, Kryvych N, McMurry J, Marioni JC, Malone J, Megy K, Rustici G, Tang AY, Taubert J, Williams E, Mannion O, Parkinson HE, and Brazma A. 2014. Expression Atlas update--a database of gene and transcript expression from microarray- and sequencing-based functional genomics experiments. Nucleic Acids Res 42:D926-932. 10.1093/nar/gkt1270

Quinlan AR, and Hall IM. 2010. BEDTools: a flexible suite of utilities for comparing genomic features. Bioinformatics 26:841-842. 10.1093/bioinformatics/btq033

Ritchie ME, Phipson B, Wu D, Hu Y, Law CW, Shi W, and Smyth GK. 2015. limma powers differential expression analyses for RNA-sequencing and microarray studies. Nucleic Acids Res 43:e47. 10.1093/nar/gkv007

Robin X, Turck N, Hainard A, Tiberti N, Lisacek F, Sanchez JC, and Muller M. 2011. pROC: an open-source package for R and $\mathrm{S}+$ to analyze and compare ROC curves. BMC Bioinformatics 12:77. 10.1186/1471-2105-12-77

Silva D, Antunes J, Balamurugan K, Duncan G, Alho CS, and McCord B. 2016. Developmental validation studies of epigenetic DNA methylation markers for the detection of blood, semen and saliva samples. Forensic Sci Int Genet 23:55-63. 10.1016/j.fsigen.2016.01.017

Song YQ, Karasugi T, Cheung KM, Chiba K, Ho DW, Miyake A, Kao PY, Sze KL, Yee A, Takahashi A, Kawaguchi Y, Mikami Y, Matsumoto M, Togawa D, Kanayama M, Shi D, Dai J, Jiang Q, Wu C, Tian W, Wang N, Leong JC, Luk KD, Yip SP, Cherny SS, Wang J, Mundlos S, Kelempisioti A, Eskola PJ, Männikkö M, Mäkelä P, Karppinen J, Järvelin MR, O'Reilly PF, Kubo M, Kimura T, Kubo T, Toyama Y, Mizuta H, Cheah KS, Tsunoda T, Sham PC, Ikegawa S, and Chan D. 2013. Lumbar disc degeneration is linked to a carbohydrate sulfotransferase 3 variant. J Clin Invest 123:4909-4917. 10.1172/jci69277

Taki T, and Kibayashi K. 2015. Characterization of cellular and extracellular DNA in saliva. Leg Med (Tokyo) 17:471474. 10.1016/j.legalmed.2015.10.003

Tay Y, Rinn J, and Pandolfi PP. 2014. The multilayered complexity of ceRNA crosstalk and competition. Nature 505:344-352. 10.1038/nature12986

Tian C, Deng Y, Jin Y, Shi S, and Bi H. 2018. Long non-coding RNA RNCR3 promotes prostate cancer progression through targeting miR-185-5p. Am J Transl Res 10:1562-1570.

Tsai TT, Lai PL, Liao JC, Fu TS, Niu CC, Chen LH, Lee MS, Chen WJ, Fang HC, Ho NY, and Pang JH. 2013. Increased periostin gene expression in degenerative intervertebral disc cells. Spine J 13:289-298.

Peer] reviewing PDF | (2020:05:48863:2:0:NEW 11 Sep 2020) 
10.1016/j.spinee.2013.01.040

Vergroesen PP, Kingma I, Emanuel KS, Hoogendoorn RJ, Welting TJ, van Royen BJ, van Dieen JH, and Smit TH. 2015. Mechanics and biology in intervertebral disc degeneration: a vicious circle. Osteoarthritis Cartilage 23:1057-1070. 10.1016/j.joca.2015.03.028

Wan ZY, Song F, Sun Z, Chen YF, Zhang WL, Samartzis D, Ma CJ, Che L, Liu X, Ali MA, Wang HQ, and Luo ZJ. 2014. Aberrantly expressed long noncoding RNAs in human intervertebral disc degeneration: a microarray related study. Arthritis Res Ther 16:465. 10.1186/s13075-014-0465-5

Wang H, He P, Pan H, Long J, Wang J, Li Z, Liu H, Jiang W, and Zheng Z. 2018a. Circular RNA circ-4099 is induced by TNF- $\alpha$ and regulates ECM synthesis by blocking miR-616-5p inhibition of Sox9 in intervertebral disc degeneration. Exp Mol Med 50:27. 10.1038/s12276-018-0056-7

Wang HQ, Yu XD, Liu ZH, Cheng X, Samartzis D, Jia LT, Wu SX, Huang J, Chen J, and Luo ZJ. 2011. Deregulated miR-155 promotes Fas-mediated apoptosis in human intervertebral disc degeneration by targeting FADD and caspase-3. J Pathol 225:232-242. 10.1002/path.2931

Wang Z, Li Y, Wang Y, Wang X, Zhang J, and Tian J. 2018b. Association between GDF5 single nucleotide polymorphism rs143383 and lumbar disc degeneration. Exp Ther Med 16:1900-1904. 10.3892/etm.2018.6382

Williams FM, Bansal AT, van Meurs JB, Bell JT, Meulenbelt I, Suri P, Rivadeneira F, Sambrook PN, Hofman A, Bierma-Zeinstra S, Menni C, Kloppenburg M, Slagboom PE, Hunter DJ, MacGregor AJ, Uitterlinden AG, and Spector TD. 2013. Novel genetic variants associated with lumbar disc degeneration in northern Europeans: a meta-analysis of 4600 subjects. Ann Rheum Dis 72:1141-1148. 10.1136/annrheumdis-2012201551

Xu YQ, Zhang ZH, Zheng YF, and Feng SQ. 2016. Dysregulated miR-133a Mediates Loss of Type II Collagen by Directly Targeting Matrix Metalloproteinase 9 (MMP9) in Human Intervertebral Disc Degeneration. Spine (Phila Pa 1976) 41:E717-724. 10.1097/brs.0000000000001375

Yuan B, Ji W, Fan B, Zhang B, Zhao Y, and Li J. 2018. Association analysis between thrombospondin-2 gene polymorphisms and intervertebral disc degeneration in a Chinese Han population. Medicine (Baltimore) 97:e9586. 10.1097/md.0000000000009586

Zaina F, Tomkins-Lane C, Carragee E, and Negrini S. 2016. Surgical versus non-surgical treatment for lumbar spinal stenosis. Cochrane Database Syst Rev:Cd010264. 10.1002/14651858.CD010264.pub2

Zhang F, Zhao X, Shen H, and Zhang C. 2016. Molecular mechanisms of cell death in intervertebral disc degeneration (Review). Int J Mol Med 37:1439-1448. 10.3892/ijmm.2016.2573

Zhang Y, Zhang R, Ding X, and Ai K. 2018. EFNB2 acts as the target of miR-557 to facilitate cell proliferation, migration and invasion in pancreatic ductal adenocarcinoma by bioinformatics analysis and verification. $\mathrm{Am}$ J Transl Res 10:3514-3528.

Zhu Y, Qiao L, Zhou Y, Ma N, Wang C, and Zhou J. 2018. Long non-coding RNA FOXD2-AS1 contributes to colorectal cancer proliferation through its interaction with microRNA-185-5p. Cancer Sci 109:2235-2242. $10.1111 /$ cas. 13632

\section{Legends of Figures}

\section{Figure 1: Differentially expressed IDD genes}

(A and B) Volcano plots represent the DEGs in the degenerative disc samples and control disc samples in the 
GSE15227 and GSE34095 datasets, respectively. (C and D) Hierarchical cluster heatmaps of the GSE15227 and GSE34095 datasets displaying the DEGs in the degenerative disc samples and the control disc samples. Blue represents the downregulated and red represents the upregulated. (E) Venn diagram of DEGs in the GSE15227 and GSE34095 datasets. The common area represents the overlapping genes. DEG, differentially expressed genes.

\section{Figure 2: Selection of optimal feature genes}

Ranking of the top 13 IDD-related genes using neural networks (A) and random forest (B). Extraction of the optimum feature genes from the 13 IDD-related genes was carried out using recursive feature elimination (C). Classification efficiency of the optimum feature genes in the model as evaluated using the ROC curve in the GSE15227 (D), GSE34095 (E), and GSE56081 (F) datasets, respectively.

\section{Figure3: Identification of target miRNAs of the optimal feature genes}

(A)Volcano plots represent the DEMs of the degenerative disc samples and control disc samples in the GSE1 16726 dataset. (B) Hierarchical cluster heatmaps of the GSE116726 dataset display the DEMs to compare degenerative disc samples and control disc samples. Blue represents the degenerative samples and red represents the control samples. (C) Venn diagram of DEMs in the GSE116726 and GSE63492 datasets. The common area represents the overlapping DEMs. (D)Venn diagram of miRNAs in overlapping DEMs and target miRNAs of optimum feature genes. The common area represents the overlapping miRNAs. DEM, differentially expressed miRNA.

\section{Figure 4: ceRNA network construction}

ceRNA network of optimum IDD feature genes with DEMs, DELs, and DECs. The blue circles represent optimum IDD feature genes, the red triangles represent DECs, the green diamonds represent DELs, and the yellow arrows represent DEMs. ceRNA, competing endogenous RNA; DEC, differentially expressed circRNA; DEL, differentially expressed IncRNA; and DEM, differentially expressed miRNA.

\section{Figure 5: Validation of optimal feature genes}

The expression levels of the four optimum feature genes in the GSE15227, GSE34095, and GSE56081 datasets, respectively. (A, B and C) The expression level of BCSA4. (D, E and F) The expression level of CRNKL1. (G, $\mathrm{H}$ and I) The expression level of SCRG1. (J, K and L) The expression level of WDR46. * represents P value $<$ $0.05, * * *$ represents $\mathrm{P}$ value $<0.001, * * * *$ represents $\mathrm{P}$ value $<0.0001$, and NS represents not significant.

\section{Table 1. Basic information of the expression profiles}

Table 2. The top three miRNAs and circRNAs related to optimal feature genes in the network

\section{Supplemental Files}

\section{Figure $S 1$ : Flow chart presentation of the study}

IDD, intervertebral disc degeneration; DEG, differential expression gene; RFE, recursive feature elimination; 
534 SVM, support vector machine; DEM, differential expression miRNA; DEL, differential expression lncRNA; 535 DEC, differential expression circRNA; ceRNA, competing endogenous RNA.

536 Figure S2: Differential expression analysis of the GSE56081, GSE63492, and GSE67566 datasets.

537 Volcano plots represent the DEGs (A) and DELs (C) of the degenerative disc samples and control disc samples, 538 respectively, in the GSE56081 dataset. Hierarchical cluster heatmaps represent the DEGs (B) and DELs (D) of 539 the degenerative disc samples and control disc samples, respectively, in the GSE56081 dataset. Hierarchical 540 cluster heatmaps represent the DEMs in the GSE63492 dataset (E) and the DECs (F) in the GSE67566 dataset. 541 Blue represents the downregulated and red represents the upregulated.

Table S1: All differentially expressed circRNAs in the GSE67566 dataset.

544 Table S2: All differentially expressed miRNAs in the GSE63492 dataset.

545 Table S3: The nodes of optimal feature genes, miRNAs, IncRNAs, and circRNAs in the ceRNA network.

Document S1: Differentially expressed mRNAs in the GSE56081 dataset.

Document S2: Differentially expressed IncRNAs in the GSE56081 dataset.

Document S3: The target miRNAs of DECs analyzed by miRanda tool.

Document S4: The target miRNAs of DELs analyzed by miRanda tool.

Document S5: Importance rankings of the 13 overlapping DEGs evaluated by random forest. 


\section{Table $\mathbf{1}$ (on next page)}

Basic information of the expression profiles 
1 Table 1. Basic information of expression profiles included in the study

2

\begin{tabular}{|c|c|c|c|c|c|}
\hline Type & Series & Platform & $\begin{array}{l}\text { Source } \\
\text { name }\end{array}$ & $\begin{array}{l}\text { Number of samples } \\
\text { (control/degenerative) }\end{array}$ & $\begin{array}{l}\text { Publication } \\
\text { year }\end{array}$ \\
\hline mRNA & GSE34095 & GPL96 & $\begin{array}{l}\text { Disc } \\
\text { tissue }\end{array}$ & $6(3 / 3)$ & 2012 \\
\hline mRNA & GSE15227 & GPL1352 & $\begin{array}{l}\text { Disc } \\
\text { tissue }\end{array}$ & $15(12 / 3)$ & 2009 \\
\hline miRNA & GSE63492 & GPL19449 & $\begin{array}{l}\text { Nucleus } \\
\text { pulposus }\end{array}$ & $10(5 / 5)$ & 2016 \\
\hline miRNA & GSE116726 & GPL20712 & $\begin{array}{l}\text { Nucleus } \\
\text { pulposus }\end{array}$ & $6(3 / 3)$ & 2018 \\
\hline $\begin{array}{l}\text { mRNA-1 } \\
\text { ncRNA }\end{array}$ & GSE56081 & GPL15314 & $\begin{array}{l}\text { Nucleus } \\
\text { pulposus }\end{array}$ & $10(5 / 5)$ & 2014 \\
\hline circRNA & GSE67566 & GPL19978 & $\begin{array}{l}\text { Nucleus } \\
\text { pulposus }\end{array}$ & $10(5 / 5)$ & 2016 \\
\hline
\end{tabular}

3

4 
Table 2 (on next page)

The top three miRNAs and circRNAs related to optimal feature genes in the network 
1 Table 2. The top 3 miRNAs and circRNAs related to optimal feature genes in the network

2

\begin{tabular}{llc}
\hline Type & name & Number of directed edges \\
\hline miRNA & hsa-miR-4728-5p & 58 \\
miRNA & hsa-miR-5196-5p & 41 \\
miRNA & hsa-miR-185-5p & 34 \\
circRNA & hsa_circRNA_100723 & 12 \\
circRNA & hsa_circRNA_104471 & 11 \\
circRNA & hsa_circRNA_100750 & 9 \\
\hline
\end{tabular}

3

4 


\section{Figure 1}

Figure 1: Differentially expressed IDD genes

( $A$ and $B$ ) Volcano plots represent the DEGs in the degenerative disc samples and control disc samples in the GSE15227 and GSE34095 datasets, respectively. (C and D) Hierarchical cluster heatmaps of the GSE15227 and GSE34095 datasets displaying the DEGs in the degenerative disc samples and the control disc samples. Blue represents the downregulated and red represents the upregulated. (E) Venn diagram of DEGs in the GSE15227 and GSE34095 datasets. The common area represents the overlapping genes. DEG, differentially expressed genes. 

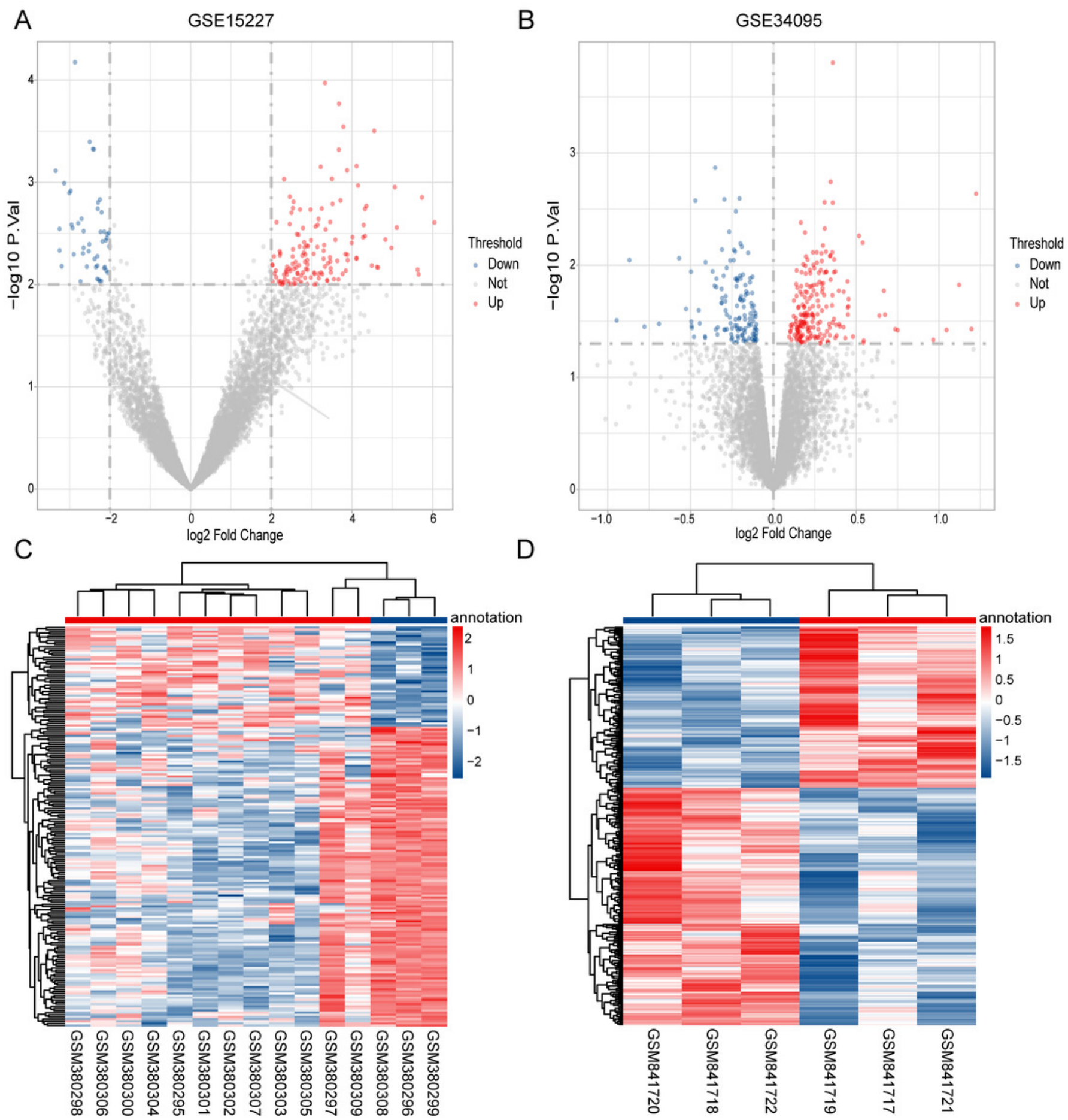

E

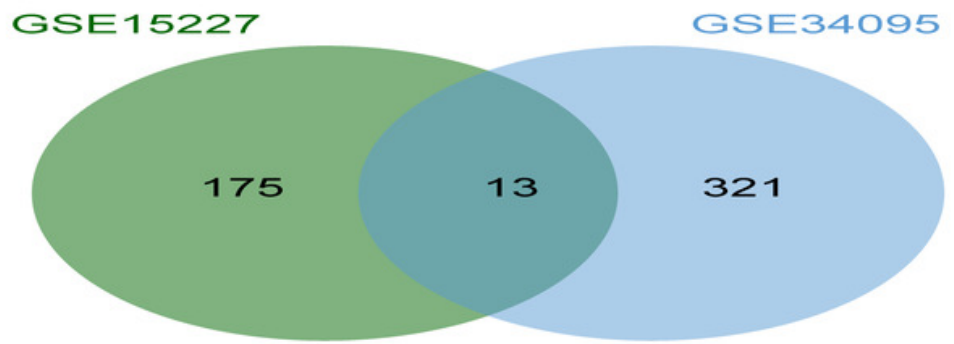




\section{Figure 2}

Figure 2: Selection of optimal feature genes

Ranking of the top 13 IDD-related genes using neural networks (A) and random forest (B). Extraction of the optimum feature genes from the 13 IDD-related genes was carried out using recursive feature elimination $(C)$. Classification efficiency of the optimum feature genes in the model as evaluated using the ROC curve in the GSE15227 (D), GSE34095 (E), and GSE56081 (F) datasets, respectively. 

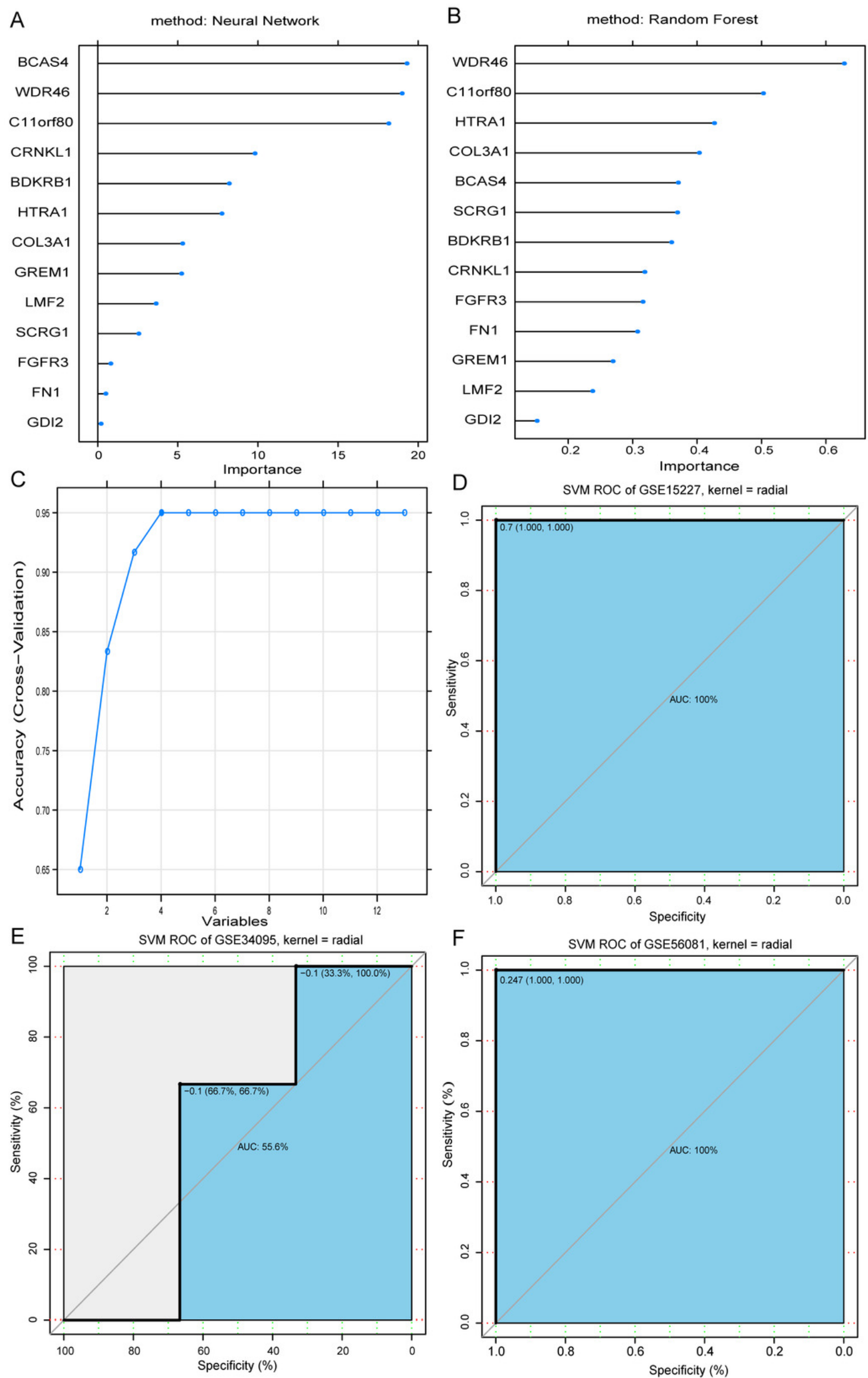


\section{Figure 3}

Figure 3: Identification of target miRNAs of optimal feature genes

(A)Volcano plots represent the DEMs of the degenerative disc samples and control disc samples in the GSE116726 dataset. (B) Hierarchical cluster heatmaps of the GSE116726 dataset display the DEMs to compare degenerative disc samples and control disc samples. Blue represents the degenerative samples and red represents the control samples. (C) Venn diagram of DEMs in the GSE116726 and GSE63492 datasets. The common area represents the overlapping DEMs. (D)Venn diagram of miRNAs in overlapping DEMs and target miRNAs of optimum feature genes. The common area represents the overlapping miRNAs. DEM, differentially expressed miRNA. 

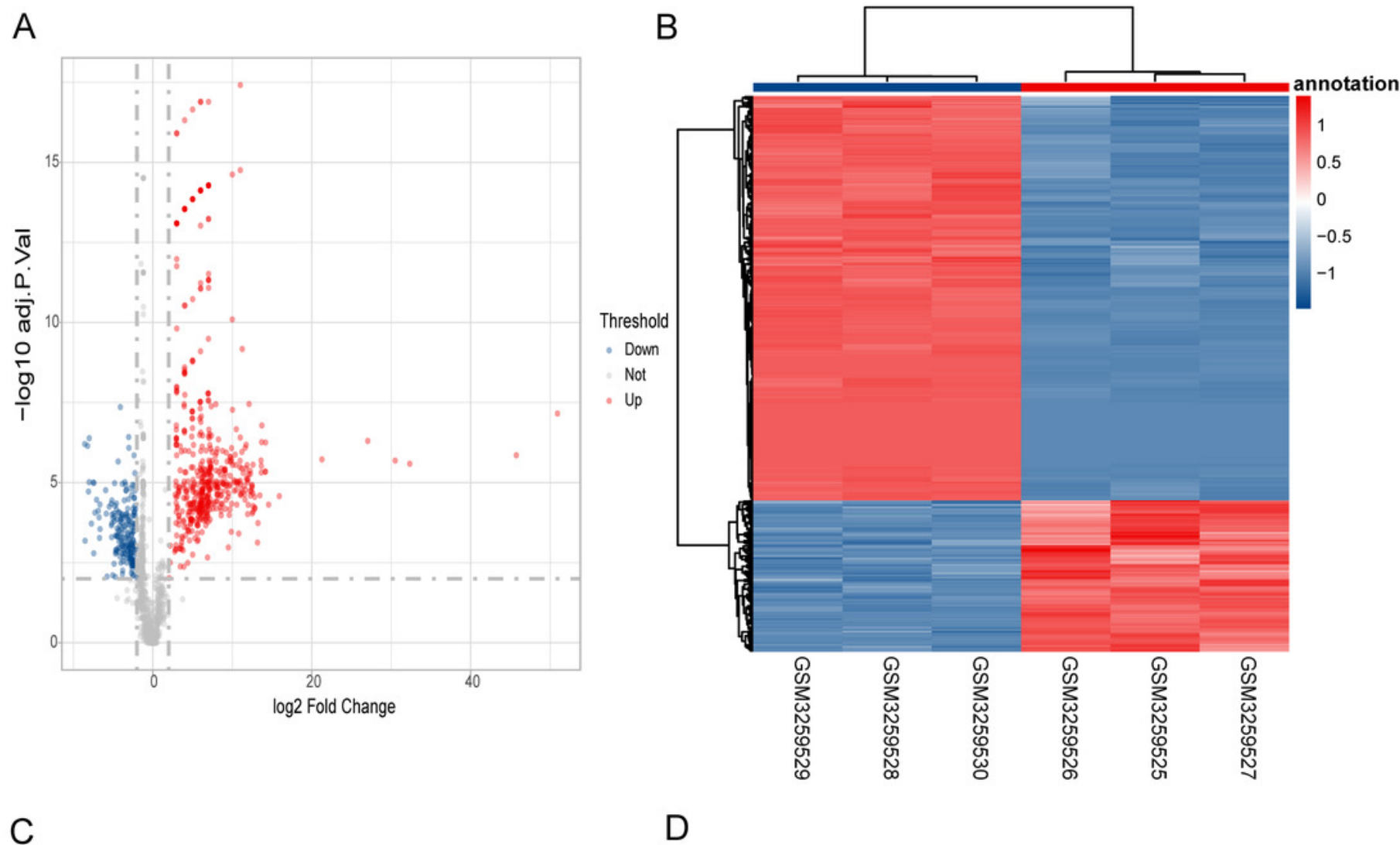

C
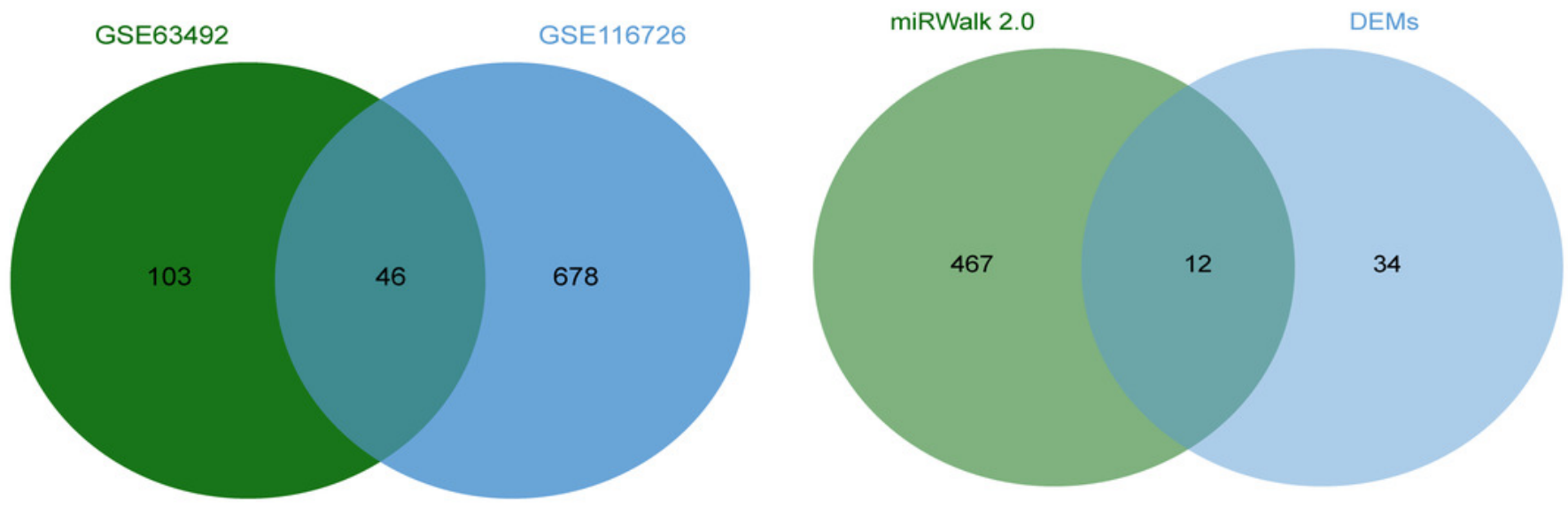


\section{Figure 4}

Figure 4: ceRNA network construction

ceRNA network of optimum IDD feature genes with DEMs, DELs, and DECs. The blue circles represent optimum IDD feature genes, the red triangles represent DECs, the green diamonds represent DELs, and the yellow arrows represent DEMs. ceRNA, competing endogenous RNA; DEC, differentially expressed circRNA; DEL, differentially expressed IncRNA; and DEM, differentially expressed miRNA. 


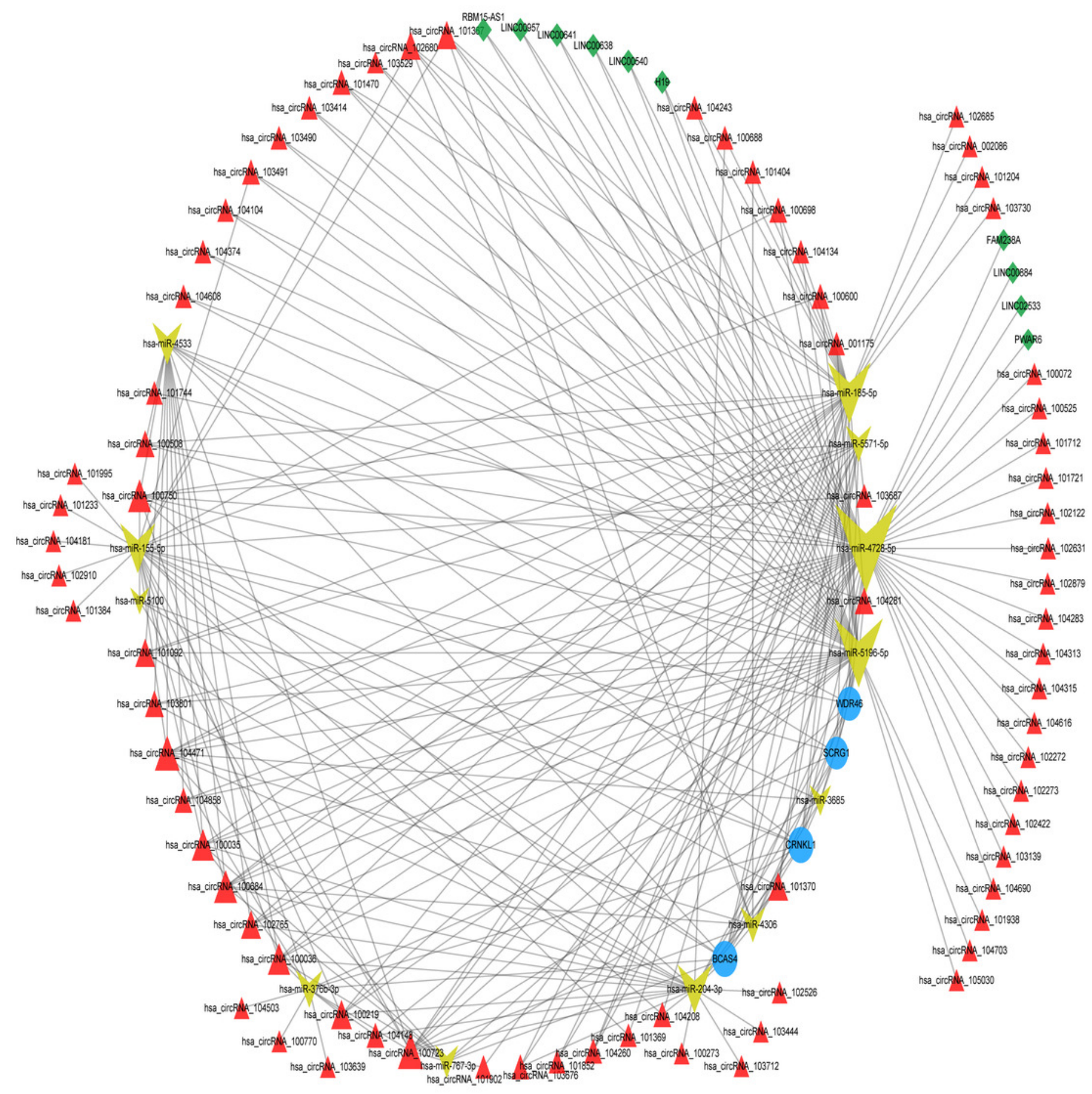




\section{Figure 5}

Figure 5: Validation of optimal feature genes

The expression levels of the four optimum feature genes in the GSE15227, GSE34095, and GSE56081 datasets, respectively. (A, B and C) The expression level of BCSA4. (D, E and F) The expression level of CRNKL1. (G, $\mathrm{H}$ and I) The expression level of SCRG1. (J, $\mathrm{K}$ and $\mathrm{L}$ ) The expression level of WDR46. * represents $\mathrm{P}$ value $<0.05$, *** represents $\mathrm{P}$ value $<0.001$, **** represents $P$ value $<0.0001$, and NS represents not significant. 
A

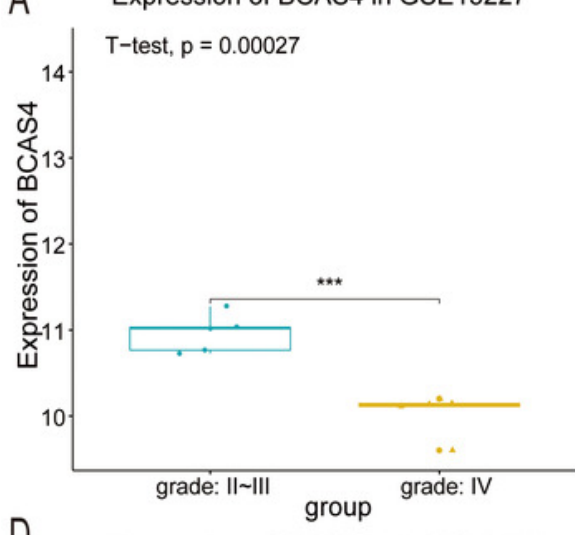

Expression of CRNKL1 in GSE15227

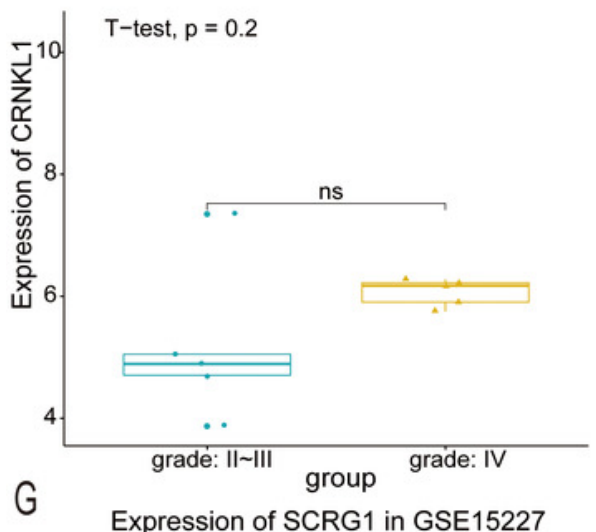

Expression of SCRG1 in GSE15227

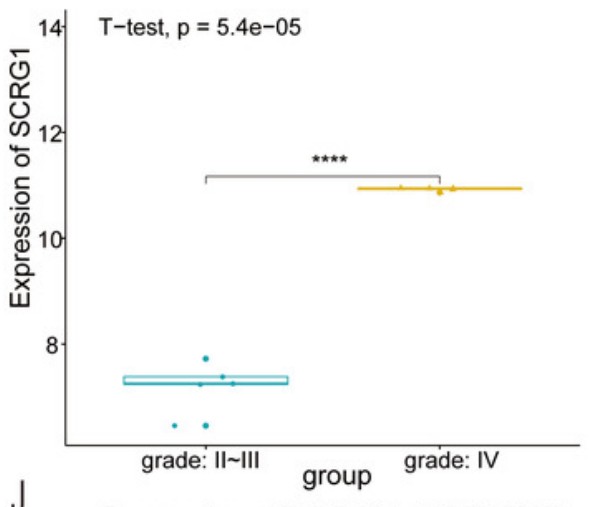

Expression of WDR46 in GSE15227

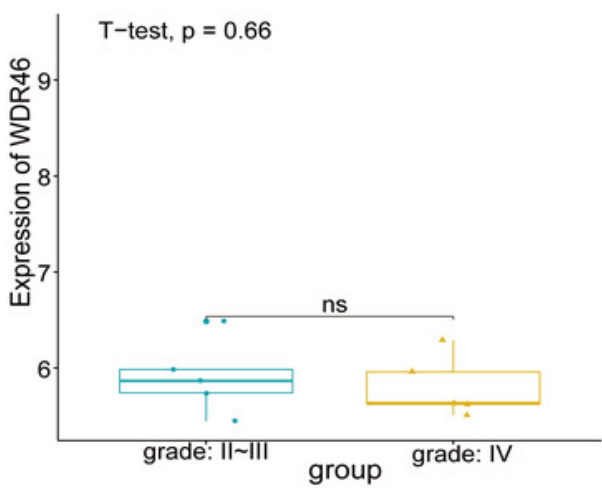

B Expression of BCAS4 in GSE34095

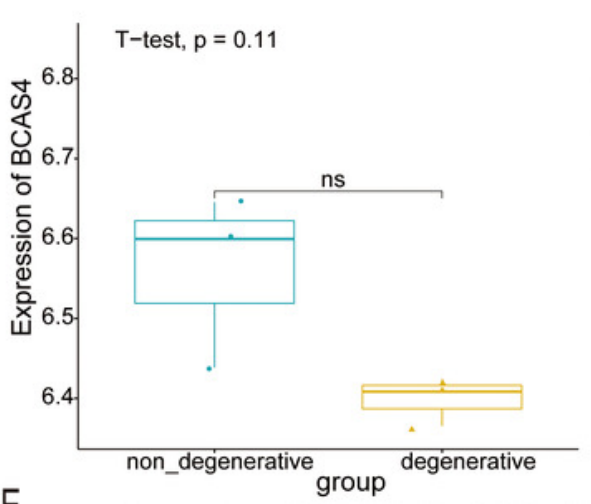

E

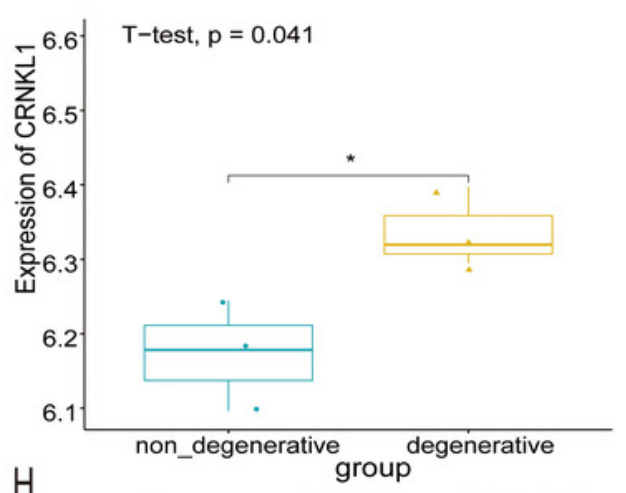

Expression of SCRG1 in GSE34095

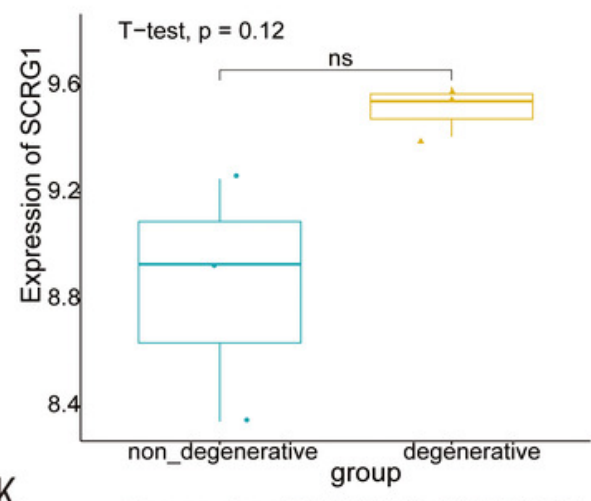

Expression of WDR46 in GSE34095

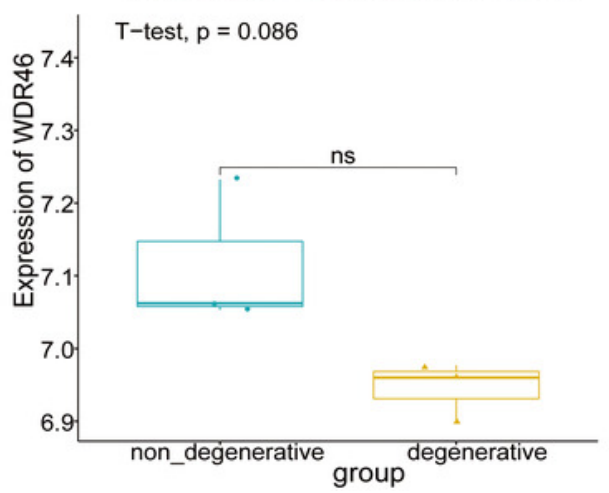

C Expression of BCAS4 in GSE56081

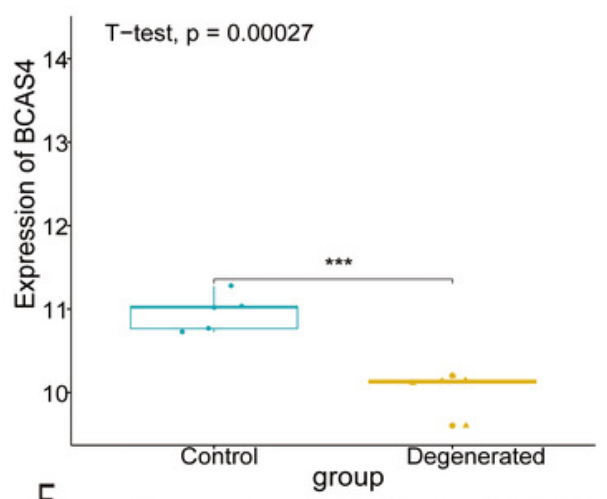

Expression of CRNKL1 in GSE56081

T-test, $p=0.2$

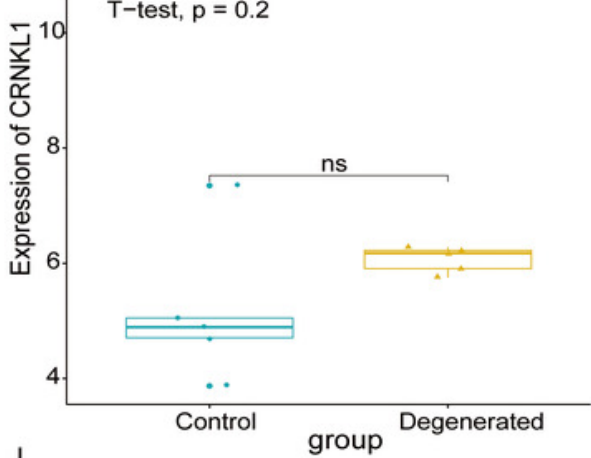

Expression of SCRG1 in GSE56081

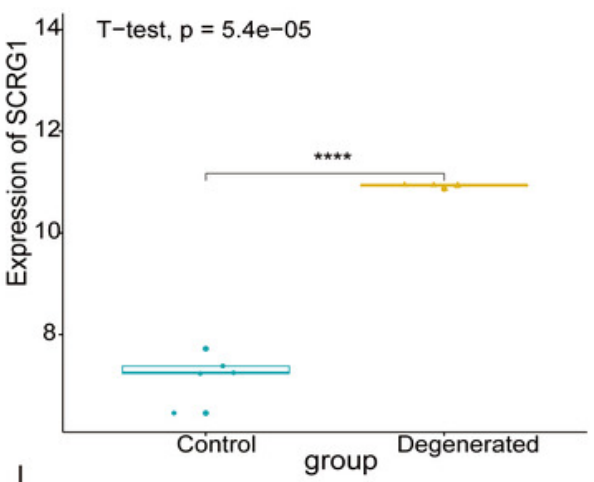

Expression of WDR46 in GSE56081

T-test, $\mathrm{p}=0.66$

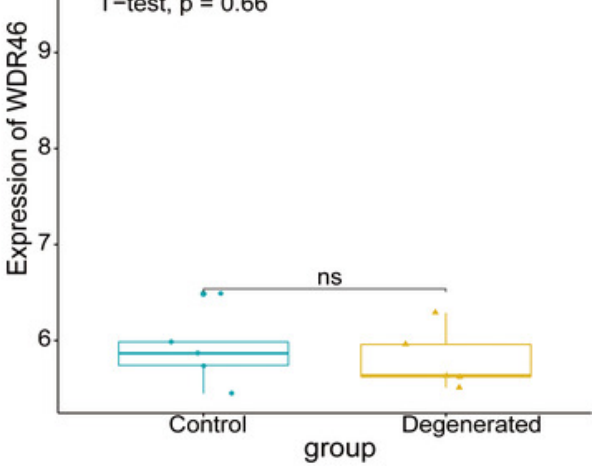

